\title{
Application of a three-microneedle device for the delivery of local anesthetics
}

\author{
This article was published in the following Dove Press journal: \\ Patient Preference and Adherence \\ 21 April 2015 \\ Number of times this article has been viewed
}

\author{
Kayoko Ishikawa' \\ Hidekazu Fukamizu' \\ Tetsuya Takiguchi' \\ Yusuke Ohta' \\ Yoshiki Tokura ${ }^{2}$ \\ 'Department of Plastic and \\ Reconstructive Surgery, Hamamatsu \\ University School of Medicine, \\ Hamamatsu, Japan; ${ }^{2}$ Department \\ of Dermatology, Hamamatsu \\ University School of Medicine, \\ Hamamatsu, Japan
}

\begin{abstract}
Purpose: We investigated the effectiveness of a newly developed device for the delivery of local anesthetics in the treatment of axillary osmidrosis and hyperhidrosis. We developed a device with three fine, stainless steel needles fabricated with a bevel angle facing outside ("three-microneedle device" [TMD]) to release a drug broadly and homogeneously into tissue in the horizontal plane. Use of this device could reduce the risk of complications when transcutaneous injections are undertaken.
\end{abstract}

Patients and methods: Sixteen Japanese patients were enrolled. The mean volume of lidocaine hydrochloride per unit area needed to elicit anesthesia when using a TMD was compared with that the volume required when using a conventional 27-gauge needle. The visual analog scale (VAS) score of needlestick pain and injection-associated pain was also compared.

Results: The mean volume of lidocaine hydrochloride per unit area to elicit anesthesia using the TMD was significantly lower than that the volume required when using the conventional 27-gauge needle. The VAS score of needlestick pain for the TMD was significantly lower than that the VAS score for the 27-gauge needle.

Conclusion: These data suggest that the TMD could be useful for the delivery of local anesthetics in terms of clinical efficacy and avoidance of adverse effects.

Keywords: three-microneedle device, transcutaneous drug delivery, local anesthesia, lidocaine, pain

\section{Introduction}

Transcutaneous injections are often used for the induction of local anesthesia. ${ }^{1}$ However, injections using a conventional needle can cause local and systemic side effects. Patients can suffer unpleasant feelings such as fear, pain, and anxiety. If the injection is carried out by unskilled physicians, the risk of adverse effects such as unexpected diffusion and inadequate placement may increase. In particular, unskilled physicians tend to inject a large volume of anesthetics under the thin dermis of axillae and eyelids. Therefore, a reliable and effective device to deliver local anesthetics that is relatively noninvasive and not painful is desired to ensure patient satisfaction.

Recently, we developed a new device with three fine, stainless steel needles ("three-microneedle device" [TMD]). These three microneedles are fabricated with a bevel angle facing the outside and can release a drug broadly and homogeneously at the target depth in tissue. ${ }^{2}$ Previously, we reported on the clinical efficacy and patient satisfaction of the TMD injections of botulinum toxin A for wrinkle reduction in patients with glabellar rhytides. In the present study, we investigated the effectiveness of this newly developed TMD for the delivery of local anesthetics for patients undergoing treatment for axillary osmidrosis and hyperhidrosis.
Correspondence: Hidekazu Fukamizu Department of Plastic and Reconstructive Surgery, Hamamatsu University School of Medicine, I-20-I Handayama Higashi-ku, Hamamatsu, 43I-3192, Japan Tel +8I 5343521 II

Fax +81534352882

Email fukamizu@hama-med.ac.jp 


\section{Patients and methods}

\section{Patients}

Sixteen Japanese patients (four males and 12 females; mean age, $27.8 \pm 12.4$ years) suffering from axillary osmidrosis with excessive malodor and/or hyperhidrosis who had not previously undergone surgical treatment were enrolled. Patients were blinded to which device was used in which axilla.

\section{Ethical approval}

This study protocol was approved by the Ethics Review Board of the Hamamatsu University School of Medicine (Hamamatsu, Japan). All volunteers and patients provided written informed consent.

\section{Device: Paskin ${ }^{\circledR}$}

We employed a newly developed device, Paskin ${ }^{\circledR}$, created by Nanbu Plastics Co, Ltd (Yoshida-cho, Japan) (Figure 1). Each stainless steel needle had an outer diameter of $0.26 \mathrm{~mm}$ (31 gauge), an exposed length of $1.5 \mathrm{~mm}$, and a bevel angle of $12^{\circ}$ (Figure 2). The efficacy and characteristics of Paskin ${ }^{\circledR}$ have been reported. ${ }^{2}$ This TMD was set on a polycarbonate pedestal with an outer diameter of $6.5 \mathrm{~mm}$ and affixed to a conventional syringe of volume $5 \mathrm{~mL}$ (Terumo Corporation, Tokyo, Japan).

\section{Control}

We used a conventional 27-gauge needle as a control. It had an outer diameter of $0.40 \mathrm{~mm}$, an exposed length of $19 \mathrm{~mm}$, and a short bevel angle of $18^{\circ}$.

\section{Assessments and clinical outcomes}

Patients with osmidrosis and/or hyperhidrosis were placed in a supine position with the arms abducted from the trunk

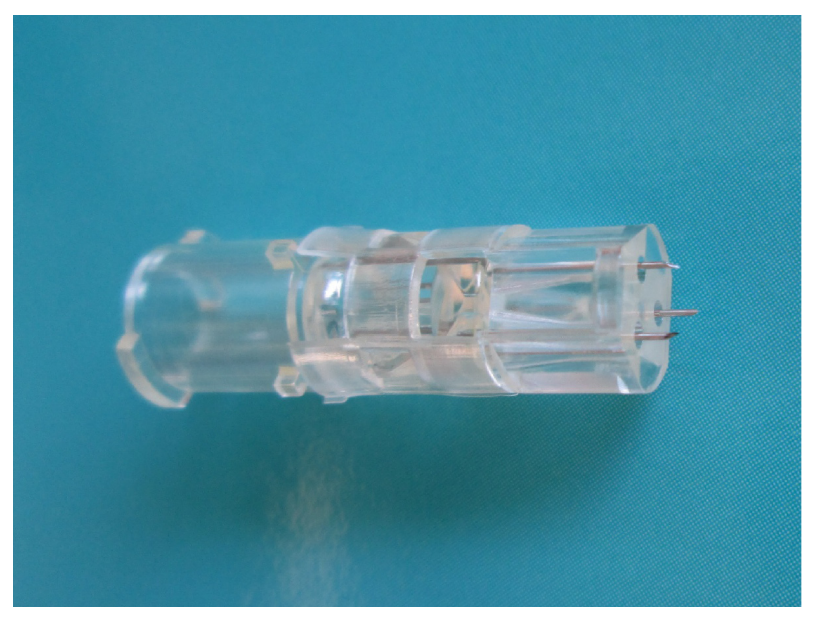

Figure I A photograph of the TMD with three fine, stainless steel needles. Abbreviation: TMD, three-microneedle device. to $\sim 100^{\circ}$ to expose the axillae and avoid injury of the brachial plexus. Markings were made around an axillary hair-bearing area.

Local anesthesia with $0.5 \%$ lidocaine hydrochloride containing epinephrine was undertaken using the TMD or a conventional needle for the right or left axilla in individual patients. The same operator used TMD in one axilla and a conventional needle in the other axilla. The volume of injected lidocaine hydrochloride was monitored. There were no specific rules about injection times, drug volume per injection, or duration of injection. The endpoint for injection was to ensure a feeling of numbness and pain relief from a pin-prick in the operation area. An operator placed the TMD in the skin and injected the anesthetic until the skin turned white and swollen. The injection volume was determined when the white area did not become any whiter and numbness was achieved.

A conventional 27-gauge needle was used as a control because it has, per injection, an almost identical cross-sectional area as the total area of three 31-gauge needles. The operator changed the direction of the conventional 27-gauge needle in the dermis and subcutaneous tissue to spread the anesthetic after the injection.

The surgical procedure was undertaken by two experienced surgeons. An endoscopic shaver (XPS Microsystem; Japan Medtronic, Tokyo, Japan) was inserted from a small incision to excise subcutaneous tissue and apocrine glands.

Assessment of pain in the needlestick area and injection of lidocaine was undertaken using a standard $100 \mathrm{~mm}$ visual analog scale (VAS). ${ }^{3,4}$ The injection volume of lidocaine hydrochloride was summed if additional anesthetic was needed during the procedure. The operation time was $\sim 50-60$ minutes per patient.

\section{Statistical analyses}

Data analysis was carried out using Microsoft Excel 2013 (Microsoft Corporation, Redmond, WA, USA) and image processing and analysis in Java (ImageJ, National Institutes of Health, Bethesda, MD, USA). A two-tailed paired Student's $t$-test was used $(P<0.05)$.

\section{Results}

We tested the efficacy of the TMD to elicit local anesthesia. Four Japanese males and 12 Japanese females (mean age, $27.8 \pm 12.4$ years) underwent treatment for axillary osmidrosis and/or hyperhidrosis. Identical anesthetic agents were injected into the right or left axilla using the TMD, and with 27-gauge needle into the other side.

The mean volume of lidocaine hydrochloride per unit area to elicit anesthesia using the TMD was significantly lower 
A

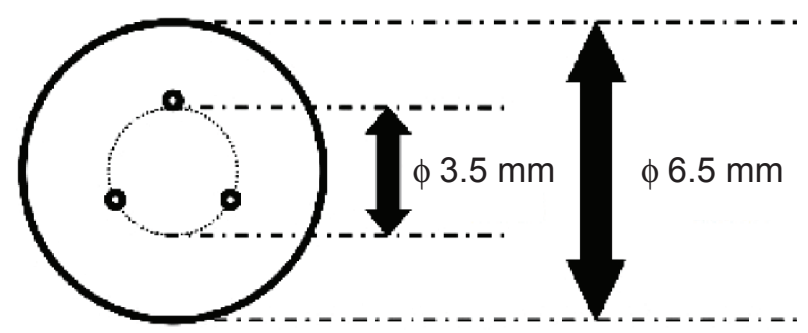

B

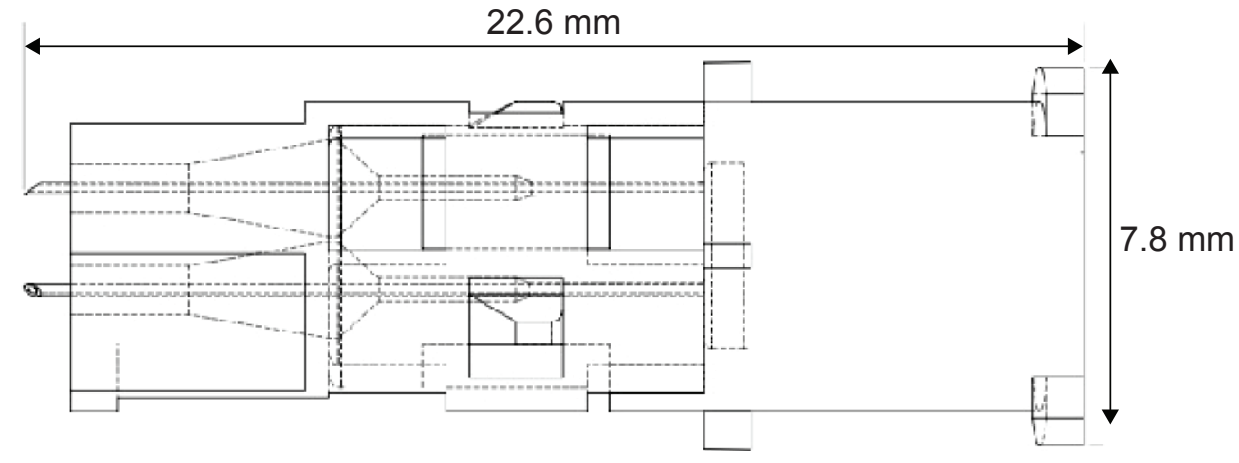

Figure 2 A structural diagram of $(\mathbf{A})$ transverse and $(\mathbf{B})$ longitudinal section of the TMD.

Notes: (A) Structural diagram of transverse section of the TMD. Three needles were set on a polycarbonate pedestal with an outer diameter of 6.5 mm. (B) Structural diagram of longitudinal section of the TMD. Each stainless steel needle had an outer diameter of $0.26 \mathrm{~mm}(3 \mathrm{I}$ gauge) and an exposed length of $\mathrm{I} .5 \mathrm{~mm}$. These three microneedles are fabricated with a bevel angle facing the outside and can release a drug broadly and homogenously.

Abbreviation: TMD, three-microneedle device.

than that with the conventional 27-gauge needle $(P<0.001)$. The VAS score of needlestick pain with the TMD was significantly lower than the VAS score obtained with the 27-gauge needle $(P=0.002)$, but injection-associated pain with the TMD was significantly higher than that with the 27-gauge needle $(P=0.01)$ (Table 1$)$. Patients were pain-free during their surgical procedure with both devices.

\section{Discussion}

We hypothesized that the bevel angle and diameter of the needle was associated with needlestick pain. Thus, the VAS score for needlestick pain with the TMD was lower

Table I Lidocaine hydrochloride injection to elicit anesthesia: volume of lidocaine and VAS scores of needlestick pain and injection-associated pain

\begin{tabular}{lll}
\hline Needle & TMD & Control (27 gauge) \\
\hline $\begin{array}{l}\text { Number of subjects } \\
\text { Injected volume }\end{array}$ & 16 & 16 \\
$\quad$ Total volume $(\mathrm{mL}) *$ & $10.9 \pm 2.73$ & $17.3 \pm 3.47$ \\
$\quad$ Injected area $\left(\mathrm{cm}^{2}\right)$ & $32.1 \pm 8.38$ & $30.0 \pm 8.06$ \\
$\quad$ Volume/area $\left(\mathrm{mL} / \mathrm{cm}^{2}\right)^{*}$ & $0.35 \pm 0.09$ & $0.60 \pm 0.14$ \\
VAS scores $(0-100 \mathrm{~mm})$ & & \\
$\quad$ Needlestick pain** & $32.5 \pm 16.8$ & $45.6 \pm 13.2$ \\
$\quad$ Injection-associated pain*** & $55.0 \pm 15.8$ & $45.9 \pm 15.5$ \\
\hline
\end{tabular}

Notes: All values are mean $\pm S D ; * P<0.001$; $* * P<0.01$; ***P $<0.05$.

Abbreviations: SD, standard deviation; TMD, three-microneedle device; VAS, visual analog scale. than the VAS score for the 27-gauge needle. Furthermore, injection-associated pain may be related to the pressure applied to the skin as well as the flow volume of the injection and duration of injection. The duration of the injection using the TMD was longer than the duration of injection for the 27-gauge needle because of its low flow volume (data not shown). We did not take any baseline VAS scores of needlestick pain and injection-associated pain for each patient. They provided VAS scores subjectively after local anesthesia.

In the treatment of axillary osmidrosis and hyperhidrosis, blood vessels and nerves around the apocrine glands should be minimally affected upon removal of apocrine glands. For effective anesthesia, needle length is a crucial parameter. Taking the thickness of the axillar dermal skin to be $1.01 \pm 0.18 \mathrm{~mm},{ }^{5}$ a TMD length of $1.5 \mathrm{~mm}$ is applicable for delivery in the hypodermal plane. Furthermore, the total volume of a given drug should be small to avoid the adverse effects of lidocaine and epinephrine. Rapid uptake of such drugs due to intravenous diffusion can result in nausea, headache, and palpitations. ${ }^{6}$

In our study, the volume of lidocaine hydrochloride delivered with the TMD was significantly lower than the volume delivered with the 27 -gauge needle. This advantage of the TMD seems to derive from effective horizontal spread of the anesthetic and fewer drainage routes in subcutaneous tissue. Comparison of drainage streams between the TMD 
and 27-gauge needles was detailed in our previous report: ${ }^{2}$ the former needle type showed several drainage streams flowing slowly from injection sites, whereas the latter needle type showed one fast-flowing drainage stream. ${ }^{2}$ The TMD is now available in six types: 31 gauge and 34 gauge in outer diameter, with $1.5 \mathrm{~mm}, 2.5 \mathrm{~mm}$, and $3.5 \mathrm{~mm}$ in exposed length. Therefore, physicians can choose the best needle for their patients.

\section{Conclusion}

Our study suggests that the TMD is useful for local anesthesia in terms of clinical efficacy and avoidance of adverse effects.

\section{Author contributions}

HF was responsible for conception and design. Authors HF, KI, TT, and YO were responsible for data acquisition. Analysis and interpretation of data were performed by HF and YT. Final approval of the manuscript was given by HF. All of the authors were responsible for drafting and revision of the manuscript.

\section{Disclosure}

The authors report no conflicts of interest in this work.

\section{References}

1. McLure HA, Rubin AP. Review of local anaethetic agents. Minerva Anestesiol. 2005;71:59-74.

2. Fukamizu H, Fujiwara M, Matsushita Y, Kim T, Tokura Y. Development of three-microneedle device for hypodermic drug delivery and clinical application. Plast Reconstr Surg. 2012;130:451-455.

3. Li X, Zhao R, Qin Z, et al. Microneedle pretreatment improves efficacy of cutaneous topical anesthesia. Am J Emerg Med. 2010;28: 130-134.

4. Gupta J, Denson DD, Felner EI, Prausnitz MR. Rapid local anesthesia in humans using minimally invasive microneedles. Clin J Pain. 2012; 28:129-135.

5. Lee Y, Hwang K. Skin thickness of Korean adults. Surg Radiol Anat. 2002;24:183-189.

6. Feldman HS. Toxicity of local anesthetic agents. Anesthetic Toxicity. 1994:107-127.
Patient Preference and Adherence

\section{Publish your work in this journal}

Patient Preference and Adherence is an international, peer-reviewed, open access journal that focuses on the growing importance of patient preference and adherence throughout the therapeutic continuum. Patient satisfaction, acceptability, quality of life, compliance, persistence and their role in developing new therapeutic modalities and compounds to optimize

\section{Dovepress}

clinical outcomes for existing disease states are major areas of interest for the journal. This journal has been accepted for indexing on PubMed Central. The manuscript management system is completely online and includes a very quick and fair peer-review system, which is all easy to use. Visit http://www. dovepress.com/testimonials.php to read real quotes from published authors. 